\title{
Probiotics Effect on the Immune System
}

Jonathan $\mathrm{Ng}$ Cho Kin

Department of Applied Sciences, University of the West of England

Correspondence:

Jonathan $\mathrm{Ng}$ Cho Kin

1001541681@student.ucsiuniversity.edu.my

jonathannck@hotmail.com

ORCID: 0000-0001-7681-8229

DOI: 10.13140/RG.2.2.14952.08962 


\section{Abstract}

Background: Probiotics can provide health benefits to an individual by regulation of the immune system. Many clinical trials have found that probiotics can prevent upper respiratory tracts infections.

Aim: To perform a systematic review and meta-analysis of available trials to investigate the effectiveness of probiotics in the prevention of upper respiratory tract infections in individuals of all ages.

Methods: A database search was carried out with databases ScienceDirect, Wiley, Springer, Medline and Pubmed. Completed randomised double blind, placebo-controlled trials were selected.

Selection criteria: Randomised placebo-controlled trials, with the objective of investigating probiotic effectiveness in preventing URTIs.

Results: Five randomised controlled trials were included, as the extracted data could be meta-analysed. This included a total of 785 participants of different age groups, children and adults. It was found that probiotics were better compared to placebo in reducing the number of participants who experienced acute upper respiratory tract infections. Probiotics were found to reduce the mean duration of acute URTI episodes, antibiotic use compared to placebo and URTI or cold related absence. Side effects and adverse effects were found to be minor and not statistically significant.

Conclusions: Probiotics were found to be better in reducing the number of participants who experienced acute URTI, the average duration per episode of acute URTI, use of antibiotics in URTI related cases and absence due to URTI. Side effects were found to be minor, making probiotics a good candidate for clinical use. This shows that probiotics 
are effective in preventing acute URTIs. However, due to limited studies and small sample size, the results are subjected to bias and should be interpreted with care.

Keywords: probiotic, upper respiratory tract infection (URTI), infection, prevention, common cold

DOI: $10.13140 / R G .2 .2 .14952 .08962$ 


\subsection{Introduction}

\subsection{History of Probiotics}

The earliest known concept of probiotics can be traced back to 1908, where Eli Metchnikoff, the Noble Prize Winner put forth a suggestion that consumption of fermented milk products or yogurt had a positive effect on the lifespan of Bulgarian peasants. The first usage of the term 'probiotic' was by Lilly and Stillwell to describe substances that was secreted by an organism and stimulate growth or had positive effect on another organism (Gupta and Garg, 2009). The World Health Organizations and Food and Agricultural Organization of the United Nations define probiotics as 'live microorganisms' which, delivers a health benefit to the host when administered in the proper amounts (K Gogineni and Morrow, 2013).

The immune system can be divided into two parts: adaptive immunity and innate immunity, both of which work together (Paul, 2011). To understand how probiotics, benefit the immune system, it is first important to be familiar with the immune system in mammals. An extensively sophisticated adaptive immune response consisting of systemic and mucosal immunity can be found in mammals, involving the interaction between different cells and molecules to provide protection against antigens and microbes. It is important to note that probiotics benefit both the systemic and mucosal immunity, which also explains how oral administration can improve many different diseases, as systemic immunity is not confined to the site of infection. Many In vitro studies done on germ free animals have successfully demonstrated that the integrity of the intestinal microbiota can affect regulation of the gut and ultimately homeostasis. This was shown to have a direct 
effect on the innate and adaptive immune development and regulation, as well as the competitive exclusion of antigens (Bienenstock et al., 2013). The following sections will explain the known effects of probiotic bacteria on the immune system, as this will enable a better understanding and allow full appreciation on the topic.

\subsection{Role of microbial species as probiotics and desirable properties}

There are many microbial species that fit the definition of probiotics, as stated by WHO. Table 1 shows a few of the important species. It was found that only lactic acid bacteria strains were of significance in nutritional benefits, namely bacteria belonging to the Lactococcus and Bifidobacterium genera (Prete et al., 2017). It is important to note that probiotic activity is strain determined, therefore, identification by genetic and phenotypic tests, such as 16SRNA sequencing that will provide a better understanding of their properties and application (Leite et al., 2015). Bacteria strains classified as probiotics need to possess certain vital characteristics and properties, to enable their beneficial effects to be carried out and be useful to the host. As probiotics have are said to mimic the normal gut microbiota, their important properties are similar. In vitro studies have determined four primary properties. Firstly, the ability to withstand acid and bile, as probiotics are administered orally and passes through the digestive system. Secondly, probiotic strains must exhibit proper adhesion to the mucosal or epithelial surface, immunological benefits of probiotics are widely dependent on this property as the prevention of pathogenic colonization by competition and immunomodulation largely depends on the colonization of probiotics or the normal gut microbiota. Thirdly, probiotic strains are expected to show antimicrobial activity. Lastly, probiotics may or may not exhibit hydrolytic bile salt activity. These characteristics, although established through 
studies, are still under scrutiny as there is a profound lack of operation standardisation in the procedures used to investigate probiotics. Because no specific set of parameters exists to describe the applications of probiotics, the relationship between target probiotic strain and physiologic function has been used in studies to establish probiotic strain characteristics (Russell, 2009).

Table 1: Microorganisms recognised as probiotics

\begin{tabular}{ll}
\hline Lactobacillus species & Bifidobacterium species \\
\hline L. acidophilus & B. adolescentis \\
\hline L. casei & B. bifidum \\
\hline L. gallinarum & B. breve \\
\hline L. rhamnosus & B. infantis \\
\hline L. reuteri & B. lactis \\
\hline L. plantarum & B. longum \\
\hline L. crispatus & B. animalis \\
\hline
\end{tabular}

\subsection{Probiotic dosage for effective health outcomes}

There is no standard dosage established for probiotics administration, most studies agree however, that dose levels showing efficacy in human studies should be used. Studies generally use a minimum concentration of $10^{6}$ colony forming units per $\mathrm{mL}(\mathrm{CFU} / \mathrm{mL})$, it is also recommended that probiotics be consumed daily for the beneficial effects (Ritchie and Romanuk, 2012). In addition to this, probiotic strains undergo commercial processing and storage, probiotic strains must retain viability and their ability to grow under such conditions (Sanders, 2008). Although viability is thought to be of importance, as probiotic functionality is dependent on cell viability for adherence and immune modulation of the 
gut, certain studies have shown that not all probiotic effects or mechanisms rely on viability. These studies have shown that certain health improvements and probiotic mechanisms are not related to viability, it is also proposed that cell wall components or the genetic material of probiotics may contribute to these health benefits. Studies propose that only the initial growth phase is important, and that viability may not be necessary during storage, as such, more care should be taken in the early production steps of probiotic supplements or products (Lahtinen, 2012).

\subsection{Probiotic mechanism of action and activity}

Probiotics have been shown by studies to exhibit many different mechanisms of actions, however, the specific way they are executed is still being studied. Based on research, probiotics are said to secrete antimicrobial peptides such as bacteriocin and aid in the maintenance of low gut $\mathrm{pH}$ levels. Probiotics also help enhance immunity by interacting with the human immune system, reinforcing the mucosal barrier, as well as competing with pathogenic bacteria for nutrients and adhesion sides (Boesten and de Vos, 2008). Immunomodulation by probiotics has been extensively researched and has provided solid evidence of the influence of probiotics in both the acquired and innate immune response, these aspects include: increased IgA secretion, phagocytosis induction, enhancing T-cell responses, including the enhancement of $T$ helper type 1 cells and the attenuation of $T$

helper type 2 (Butel, 2014). Studies have shown the correlation between airway hyperreactivity and Th2 responses, as T helper type 2 cells are responsible for allergic responses, the intervention of probiotics may be beneficial in the treatment of asthma, upper respiratory tract infections and inflammatory disorders (Xiong et al., 2012). 


\subsection{Probiotics effect on the mucosal immune system}

The lining of the gastrointestinal tract, respiratory tract and genitourinary tract surfaces are mucosal, it is also the site where antigens and pathogenic agents or bacteria are encountered (Pommerville, Alcamo and Alcamo, 2013). The digestive mucosa contains the GALT (gut associated lymphoid tissue), which consists of the Payer's patch found in the small intestine, immune responses are induced here. It is noteworthy that the adaptive immune system of mammals, including the systemic and mucosal immune system are complex. They are both linked and therefore, shows the relationship between the local mucosal immunity and the upper respiratory tract. The digestive mucosa also contains lymphoid aggregates and lymph nodes (Izcue, Coombes and Powrie, 2009). The mucosal immune system is comprised of the physical mucus barrier, antimicrobial peptides and cellular components that work in unity to prevent the body from pathogenic invasion. The mucosal epithelial cells form a physical barrier, which also serves as a site for normal microbiota colonization and is responsible for the initiation of immune responses (Koch and Nusrat, 2012). Probiotics were found to intervene in the host's mucosal immune system by secreting immunomodulins that contribute to cell signaling in the cell, this is possible by first adhering and colonizing the mucosal surface of the gut (Hemaiswarya et al., 2013). Studies on mouse intestinal loop models of mice have shown that entry of probiotics in the Peyer's patch was controlled by their interaction with IgA antibodies and have a direct effect on immune cells such as dendritic cells (Rol et al., 2012). Probiotics bacteria were also found to enhance and up-regulate dendritic cells as well as T cells, in association with an increase in cytokine TGF beta and interleukin 10 (Bienenstock et al., 2013). 


\subsection{Probiotic benefits and effects on secretory immunoglobulin A and}

\section{immunoglobulin A secreting cells}

B cells can differentiate into effector cells or plasma cells in lamina propria located in the gut, these plasma cells secrete $\lg A$ antibodies. $\lg A$ antibodies are essential and have multiple roles in the mucosal immune system, secretory $\lg A$ prevents the binding of various pathogens such as bacteria, virus and fungi. In addition to the inhibition of entry of opportunistic pathogens through the gut, $\lg A$ also has a role in the homeostasis of host and normal microbiota (Corthésy, 2013). Secretory IgA can downregulate inflammation, carry out binding to antigen-IgA complexes and remove antigen-antibody complexes (Corthésy, 2013). Two studies, Ya et al., (2008) and (Tobita, Yanaka and Otani, 2010) showed that Lactobacillus casei Zhang and Lactobacillus crispatus KT-11 respectively were able to increase intestinal fluid secretory $\lg A$ levels dose dependently, thus improving the gut mucosal immune system and resistance to pathogens. This proves that probiotic benefits the immune system via IgA secretion. A milk drink with combination of probiotics Lactobacillus casei DN-110041, Lactobacillus delbrueckii and Streptococcus thermophilus, administered daily for 3 months were also found to increase IgA producing cells in both the small and large intestines, and the bronchus (de Moreno de LeBlanc et al., 2008). Probiotics in the gut can also influence sites that are distant, IgA producing cells were found to migrate into the lymphatic circulation via the thoracic duct (de Moreno de LeBlanc et al., 2008). Probiotics have been proven to stimulate dendritic cells and epithelial cells to produce molecules that curate $\lg A$ class switching, and are $T$ cell independent, such as TGF beta or 'A Proliferation Inducing Ligand' molecule (Clemente et al., 2012). 


\subsection{Why this review is important}

Although the exact mechanisms through which probiotics confer health benefits are still largely unclear, the administration of probiotics show a positive relationship with respect to immunomodulation. There is limited data and evidence available from systematic reviews on probiotics, many show the benefits of probiotics in the treatment of infectious diarrhoea, antibiotic associated diarrhoea and pregnancy associated vaginal infections (Bernaola Aponte et al., 2013). The first study that showed the beneficial relationship between probiotics and upper respiratory tract infections showed that mice were protected from influenza after the application of Bifidobacterium breve strain TIT4064 (Yasui, 1999). Following this, research was conducted on children in day care centres in Finland, where children were given Lactobacillus rhamnosus GG during the winter season (Hatakka, 2001). However, a later study showed that probiotics had noeffect on upper respiratory infections (Hatakka, 2007). As the popularity and consumption of probiotic food products increase, it is essential that more research is done to understand the mechanisms of probiotics on URTI and the side effects to humans, if any.

\subsection{Objective}

To investigate the effectiveness of probiotics compared to an equivalent placebo in the prevention of upper respiratory tract infection in individuals of all ages. 


\subsection{Methodology}

This section will discuss and explain the process, as well as steps taken in searching for literature, journals and papers for the dissertation paper. Many scientific fields have great demand for literature reviews and meta analyses. This need is due to the constantly increasing amount of scientific publications (Rapple, 2011). A study comparing papers in 1991 to 2008 found that, there were forty times more papers in the Web of Science on biodiversity (Pautasso, 2010). It was also mentioned that although recognition is usually from primary research, literature reviews can often lead to new insights and must be compiled in a professional way for reviews to be useful (Hampton and Parker, 2011).

Before beginning a search for resources, a topic for the dissertation was chosen based on the criteria stated by Maier (2013), stating the topic must at least be interesting to the individual, be an important aspect of the field as this ensures sufficient material to write on, and is a well-defined issue. Probiotics and the effects on the immune system chosen after reading several journals and as it fits the criteria listed.

Firstly, studies were collected by conducting a search of various appropriate electronic databases. ScienceDirect and Springer were the databases chosen to be used to source and collect primary studies, research papers and journal articles. The search was carried out with key words by using the University of the West of England (UWE) library service available to students, using results obtained from the databases ScienceDirect and Springer to identify suitable research papers and studies. The full list of key words and terms used in the search can be found in Appendix A. These search items or key words were recorded and kept track of to ensure that the search can be replicated again if 
needed, (Maggio, Tannery and Kanter, 2011). A list of papers whose pdfs were unobtainable were also kept, which could be retrieved by alternative methods later (Pautasso, 2010). A few early exclusion criteria were also applied to filter and select relevant studies, these researches done in the recent 10 years to ensure up to date results in the meta-analysis. Only research done in the last 10 years and on human subjects were considered.

To enhance search specificity and result quality, Boolean logic was used to refine searches as recommended by Adorno, Garbee and Marix, (2016). The Boolean operators of 'AND' and 'OR' were utilised when performing electronic database searches. The operator 'OR' was used to obtain a wider search when identifying studies that included different probiotic strains in a single study, to solve the problem of lack of studies in the initial search. The 'AND' operator was also used for a more focused search on probiotics as intervention and illness, this enabled a more specific search for trials that included patients suffering from upper respiratory tract infections and the use of probiotics as the intervention.

The snowball sampling technique was also used, where the list of studies and references were further analysed to yield even more studies as explained by Sedgwick, (2013). The authors of the studies were also scrutinised to obtain previous work on the subject. This was useful in the electronic database search and aided in enhancing and widening the search results. A few more studies were identified by this technique and used in the metaanalysis. 
Over 20 journal articles and studies were reviewed, with a total of 5 studies selected for the final review and meta-analysis. All studies were randomised, double blind and placebo controlled, as this is a systemic review, study data was already available, and no ethics committee was required. As no experimental work was carried out on humans or animals in producing this systemic review, no consent was required. 


\subsection{Results and Discussion}

\subsection{Included studies}

20 full clinical trial texts were identified, all of which were randomized controlled trials and included probiotic supplementation as the intervention. Out of the 20 trials, eight were excluded as they were published more than 10 years ago, this was done to ensure research viability and accuracy. Three were excluded as the text was not available in English. Another four were excluded as the measured outcomes differed or was not related to this review, the extracted data would not be able to be used in the statistical analyses. Five clinical trials (Fujita 2013, Hojsak 2010a, Smith 2013, Rerksuppaphol 2012, Rautava 2009) were included, data were extracted from these five studies.

\subsection{Study design}

All five randomised controlled trials (Fujita 2013, Hojsak 2010a, Smith 2013, Rerksuppaphol 2012, Rautava 2009) are of a two-arm parallel design, the treatment arm being probiotic supplements and the control arm an equivalent placebo.

\subsection{Participants}

Two trials (Smith 2013, Fujita 2013) had adult participants aged 18 to 65 years old. The remaining three trials (Hojsak 2010, Rautava 2009, Rerksuppaphol 2012) had children participants, the exact age range was not disclosed. The country of origin of these studies are Finland (Rautava 2009), United States (Smith 2013), Croatia (Hojsak 2010), Thailand (Rerksuppaphol 2012) and Japan (Fujita 2013). 


\subsection{Interventions}

Different probiotic strains were administered, some administered a combination of two strains. The strains used were Lactobacillus rhamnosus GG, Lactobacillus acidophilus, Bifidobacterium bifidum, Bifidobacterium lactis BB-12, Bifidobacterium animalis and Lactobacillus casei Shirota. Two trials (Hojsak 2010, Fujita 2013) were administered with dairy based foods. (Smith 2013) administered in a powder form. Two trials (Rautava 2009, Rerksuppaphol 2012) administered in a capsule form. A combination of two probiotic strains were used in three trials (Smith 2013, Rerksuppaphol 2012, Rautava 2009), One strain used in two trials (Hojsak2010, Fujita 2013).

\subsection{Measured outcomes}

Although different primary outcomes were measured in the trials, a meta-analysis was possible as all had reported the number of acute URTI cases. Other outcome measures reported include: duration of acute URTI episodes, rate ratio of acute URTI episodes (Fujita 2010), antibiotic use reports (Hojsak 2010, Rautava 2009, Rerksupapphol 2012), side effects of vomiting, diarrhoea and bowel irritation (Rautava 2009, Rerksupapphol 2012, Smith 2013), time away from school due to common cold (Rerksupapphol 2012). (Hojsak 2010) reported the number of days absent from day-care due to infections, although vaguely stated with no explanation of what infections these were. 


\subsection{Risk of bias assessment for the chosen studies}

The 'Cochrane risk of bias tool' developed specifically for randomised controlled trials was used to judge and rate each study according to the Cochrane's seven criteria. The seven criteria being random generation of sequence, allocation concealment, blinding of participants and personnel, blinding of outcome assessment, incomplete outcome data, selective reporting and other bias. Graphical representations of the 'risk of bias' analysis for chosen studies are presented in figure 2 and figure 3 below.

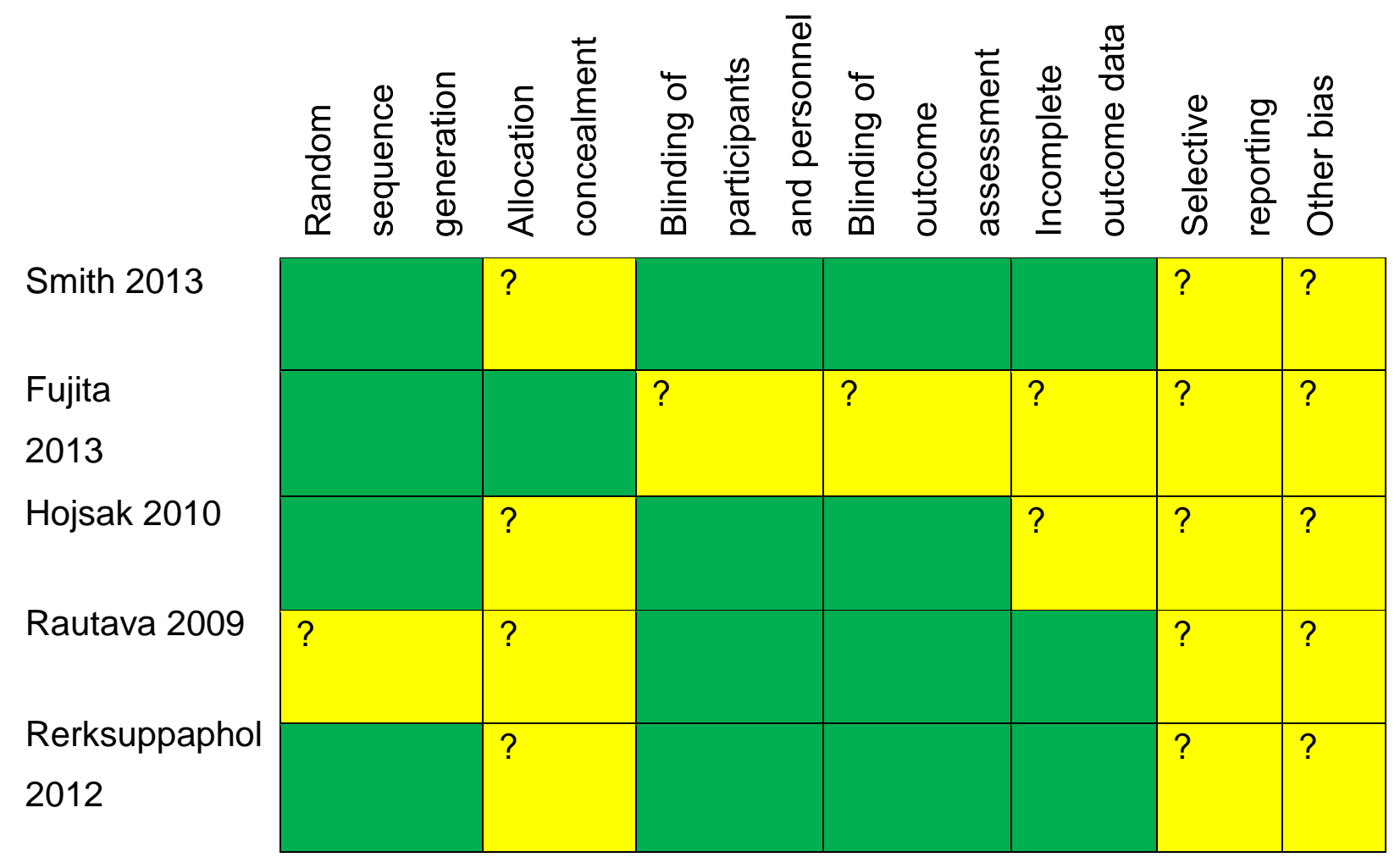

Figure 2: Summary of 'Risk of bias', checklist of bias risk review for each study 


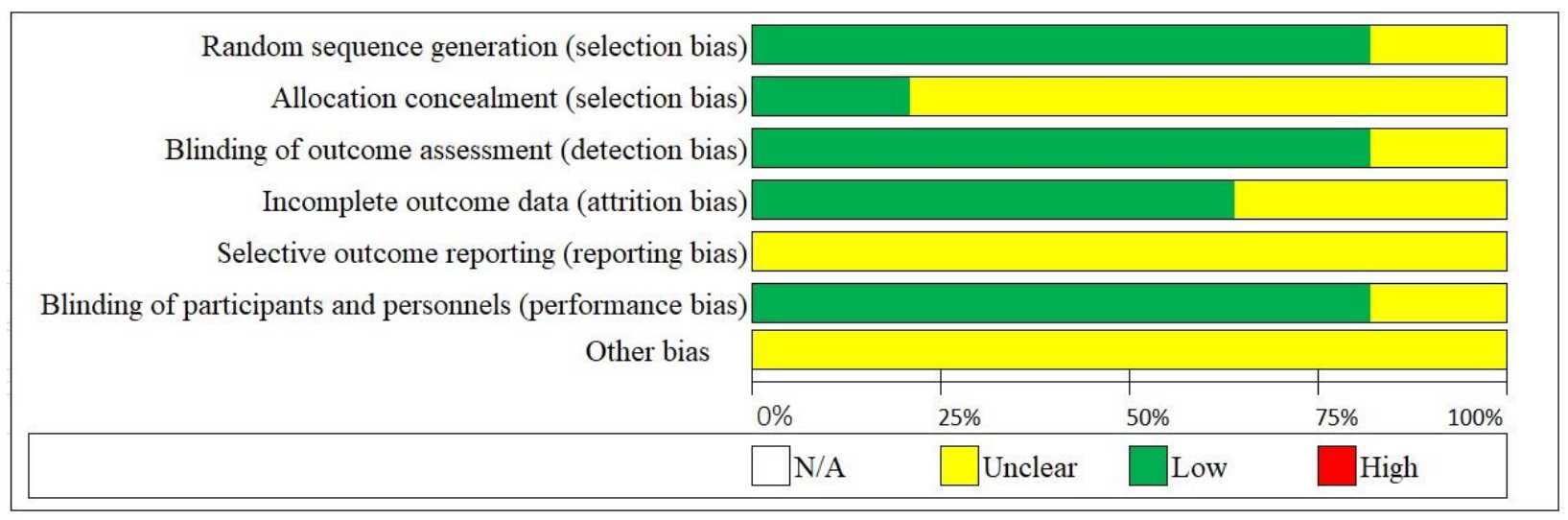

Figure 3: 'Risk of bias' graph: Risk of bias of each methodological domain represented in percentage.

\subsubsection{Random sequence generation and allocation concealment}

Four studies explained clearly the methods used in sequence generation (Fujita 2013, Hojsak 2010a, Smith 2013, Rerksuppaphol 2012). The sequence generation was not stated for Rautava (2009). Allocation concealments were not adequately described by four studies (Hojsak 2010a, Smith 2013, Rerksuppaphol 2012, Rautava 2009) studies to be conclusive. Fujita (2013) had clearly stated evidence for ruling out allocation concealment bias.

\subsubsection{Blinding of participants and personnel, blinding of outcome assessment}

All five studies were stated to be randomised double-blinded (Fujita 2013, Hojsak 2010a, Smith 2013, Rerksuppaphol 2012, Rautava 2009). Four studies provided detail descriptions of the blinding methods towards participants, personnel and outcome assessment (Hojsak 2010a, Smith 2013, Rerksuppaphol 2012, Rautava 2009). 


\subsubsection{Selective reporting}

Due to the inability of obtaining the protocols used for the studies, not enough data was available to assess the bias of selective reporting.

\subsubsection{Other bias}

Three studies (Fujita 2013, Hojsak 2010a, Rerksuppaphol 2012) had samples sizes that are small, which may possibly lead to other bias.

\subsection{Outcomes}

\subsubsection{Primary outcomes}

The number of participants who experienced acute URTI

Table 2: Chi-Square Test for number of participants with acute URTI

\begin{tabular}{|c|c|c|c|c|c|}
\hline & Value & df & $\begin{array}{l}\text { Asymp. Sig. } \\
\text { (2-sided) }\end{array}$ & $\begin{array}{l}\text { Exact Sig. } \\
\text { (2sided) }\end{array}$ & $\begin{array}{l}\text { Exact Sig. } \\
\text { (1sided) }\end{array}$ \\
\hline Pearson Chi-Square & $12.297^{a}$ & 1 & .000 & & \\
\hline Continuity Correction & 11.782 & 1 & .001 & & \\
\hline Likelihood Ratio & 12.333 & 1 & .000 & & \\
\hline Fisher's Exact Test & & & & .000 & .000 \\
\hline
\end{tabular}

$\mathrm{N}$ of Valid Cases

785

The Pearson chi-square test was used as the data obtained was categorical, this was done to test if intervention and URTI are dependent to each other. The results (Table 2) from the test are significant as the $p$-value $=0.000<0.05$, indicating that intervention and URTI incidence are dependent to each other. The null hypothesis stating that the 
variables are independent to each other is rejected, the alternative hypothesis is accepted, there is significant difference between intervention and URTI. The value of the chi-square statistic is 12.297 with $\mathrm{d} . \mathrm{f}=1$. The test is assumed to have a minimum expected count of 141.36 , which is greater than 5 . The chi-square test is assumed to be accurate. As the chi-square test does not tell if probiotics is beneficial in preventing URTI, an expected count crosstabulation table (table 3) was generated to compare the expected number of URTI cases placebo and probiotic groups.

Table 3: Intervention * URTI Crosstabulation

\begin{tabular}{|c|c|c|c|c|c|}
\hline & & & \multicolumn{2}{|c|}{ URTI } & \multirow[b]{2}{*}{ Total } \\
\hline & & & No & Yes & \\
\hline \multirow[t]{6}{*}{ Intervention } & Placebo & Count & 121 & 276 & 397 \\
\hline & & Expected Count & 144.6 & 252.4 & 397.0 \\
\hline & & $\%$ within Intervention & $30.5 \%$ & $69.5 \%$ & $100.0 \%$ \\
\hline & Probiotic & Count & 165 & 223 & 388 \\
\hline & & Expected Count & 141.4 & 246.6 & 388.0 \\
\hline & & $\%$ within Intervention & $42.5 \%$ & $57.5 \%$ & $100.0 \%$ \\
\hline \multirow[t]{3}{*}{ Total } & & Count & 286 & 499 & 785 \\
\hline & & Expected Count & 286.0 & 499.0 & 785.0 \\
\hline & & $\%$ within Intervention & $36.4 \%$ & $63.6 \%$ & $100.0 \%$ \\
\hline
\end{tabular}

From table $3,69.5 \%$ of individuals from the placebo group experienced URTI, compared to a slightly lower $57.5 \%$ from the probiotics group. The number of individuals from placebo group who experienced URTI is also higher than expected count by 24 individuals. The number of individuals from probiotic group who experienced URTI were lower than the expected count by 23 . Probiotic is shown to be statistically better in the 
primary outcome measure of preventing acute URTI based on the studies chosen in this review.

Mean duration per episode of acute URTI

Table 4: Summary of results of intervention and acute URTI episode mean duration

\begin{tabular}{|c|c|c|c|c|}
\hline & Probiotic $(\mathrm{n})$ & Mean (days) & Placebo (n) & Mean (days) \\
\hline Smith (2013) & 101 & 5.58 & 96 & 7.11 \\
\hline Fujita (2013) & 76 & 3.71 & 78 & 5.40 \\
\hline
\end{tabular}

A summary of two studies that measured the mean duration of each acute URTI case is presented in table 4. The mean duration of acute URTI for both Smith (2013) and Fujita (2013) are 5.58 and 3.71 days respectively for probiotics, with placebo being 7.11 and 5.40 days respectively. The $p$ values of the mean duration of each study is $p=0.001$ (Smith, 2013) and $p=0.037$ (Fujita, 2013), indicating significant difference between the intervention groups and mean duration of acute URTI episodes. From the results, the mean days of illness for probiotic is lower than the placebo mean by 1.53 days for (Smith, 2013) and 1.69 days for (Fujita, 2013). Although probiotics are shown to be beneficial in both these studies, further research is needed to be done with a larger sample size and different population. As the data was obtained from adult and elderly population, bias might also affect this result as the sample size was small. 


\subsubsection{Secondary outcomes}

Number of participants that used antibiotics

Table 5: Chi-square for number of participants that used antibiotics

\begin{tabular}{|c|c|c|c|c|c|c|}
\hline \multirow[b]{2}{*}{ Pearson ChiSquare } & \multirow{2}{*}{$\frac{\text { Value }}{4.809^{\mathrm{a}}}$} & \multirow[t]{2}{*}{$\mathrm{df}$} & \multicolumn{4}{|c|}{$\begin{array}{l}\text { Asymp. Sig. (2- Exact Sig. (2- Exact Sig. } \\
\begin{array}{c}\text { Exided) } \\
\text { sided) }\end{array} \\
\text { (1-sided) }\end{array}$} \\
\hline & & & 1 & .028 & & \\
\hline $\begin{array}{l}\text { Continuity } \\
\text { Correction }^{b}\end{array}$ & 4.301 & & 1 & .038 & & \\
\hline Likelihood Ratio & $\underline{4.847}$ & & 1 & .028 & & \\
\hline $\begin{array}{l}\text { FISner's Exact } \\
\text { Test }\end{array}$ & & & & & .032 & .019 \\
\hline $\mathrm{N}$ of Valid Cases & $\underline{442}$ & & & & & \\
\hline
\end{tabular}

a. 0 cells $(0.0 \%)$ have expected count less than 5 . The minimum expected count is 43.20 .

The Pearson chi-square test was used again to test the significant difference between intervention and number of participants that used antibiotic. Table 5 shows the $p$-value $=0.000<0.05$. The null hypothesis is rejected, and the alternative hypothesis is accepted, there is significant difference between intervention and antibiotic usage. The value of the chi-square statistic is 4.809 with d. $f=1$. The test is assumed to have a minimum expected count of 43.20 , which is greater than 5 . The chi-square test is assumed to be accurate. 
Table 6: Intervention * Antibiotic Crosstabulation

\begin{tabular}{|c|c|c|c|c|c|}
\hline & & & \multicolumn{2}{|c|}{ Antibiotic } & \multirow[b]{2}{*}{ Total } \\
\hline & & & No & Yes & \\
\hline \multirow[t]{6}{*}{ Intervention } & \multirow[t]{3}{*}{ Placebo } & $\underline{\text { Count }}$ & $\underline{171}$ & 54 & 225 \\
\hline & & \multirow{2}{*}{$\frac{\text { Expected Count }}{\% \text { within Intervention }}$} & $\underline{180.2}$ & 44.8 & 225.0 \\
\hline & & & $76.0 \%$ & $24.0 \%$ & $100.0 \%$ \\
\hline & \multirow[t]{3}{*}{ Probiotic } & $\underline{\text { Count }}$ & $\underline{183}$ & 34 & 217 \\
\hline & & Expected Count & 173.8 & 43.2 & 217.0 \\
\hline & & $\%$ within Intervention & $84.3 \%$ & $15.7 \%$ & $100.0 \%$ \\
\hline \multirow[t]{3}{*}{ Total } & & $\underline{\text { Count }}$ & $\underline{354}$ & 88 & 442 \\
\hline & & \multirow{2}{*}{$\frac{\text { Expected Count }}{\% \text { within Intervention }}$} & $\underline{354.0}$ & 88.0 & 442.0 \\
\hline & & & $80.1 \%$ & $19.9 \%$ & $100.0 \%$ \\
\hline
\end{tabular}

Table 6 shows the results of antibiotic use per acute URTI case, extracted and combined from three studies (Hojsak 2010, Rautava 2009, Rerksupapphol 2012). There were 217 participants of the probiotic group and 225 of the placebo group. The number of participants who used antibiotics were higher than the expected count in the placebo group, the probiotic group had a lower count of antibiotic use compared to the expected value. This shows that probiotics are statistically better in reducing the use of antibiotics in the clinical studies looked at, however, a larger sample size is needed to eliminate possible bias.

Adverse side effects

Three out of five trials had reported only minor adverse outcomes or side effects from probiotic intervention. Vomiting, excess flatulence and bowel irritability were among the 
gastrointestinal symptoms reported by Rautava, (2009), this study used Lactobacillus rhamnosus, and Bifidobacterium lactis. Adverse outcomes reported by the other two studies (Smith 2013, Rerksupapphol 2012) included all reported by Rautava, (2009), in addition to diarrhoea, nausea and soft stools. No study data was extracted from Rautava, 2009, as the adverse outcomes were only lightly and vaguely discussed. Data was extracted from the two other studies (Smith 2013, Rerksupapphol 2012), a chi-square analysis (table 7) was done to test the dependence of adverse side effects on intervention. The combined study data included 217 participants from the probiotic group and 225 from placebo group, 34 and 54 participants had experienced side effects for probiotic and placebo group respectively.

Table 7: Chi-square test for adverse side effects

\begin{tabular}{llllll}
\hline & Value & df & $\begin{array}{l}\text { Asymp. Sig. } \\
(2 \text {-sided })\end{array}$ & $\begin{array}{l}\text { Exact Sig. } \\
(2 \text { sided })\end{array}$ & $\begin{array}{l}\text { Exact Sig. } \\
(1 \text { sided })\end{array}$ \\
\hline Pearson Chi-Square .303a & 1 & .582 & & \\
\hline $\begin{array}{l}\text { Continuity } \\
\text { Correctionb }\end{array}$ & .177 & 1 & .674 & & \\
Likelihood Ratio & .303 & 1 & .582 & & .337 \\
\hline Fisher's Exact Test & & & & .607 &
\end{tabular}

\section{$\mathrm{N}$ of Valid Cases 278}

a. 0 cells $(0.0 \%)$ have expected count less than 5 . The minimum expected count is 42.87.

The $p$-value obtained from the chi square test is $p=0.582>0.05$. The $p$-value shows that the intervention is not dependent on the side effects or adverse outcomes experienced by the participants, the null hypothesis is accepted and proves the adverse effects experienced throughout the intervention are not statistically significant between the 
probiotic and placebo groups. This shows that probiotics are safe to consume and can be used clinically to prevent URTI occurrence without serious adverse side effects, this can also decrease the usage of antibiotics and antibiotic resistance. Once again due to the small sample size possible bias might arise from the results, more studies need to be done with a larger population with a combination of different probiotic strains to fully validate the side effects of probiotics.

The time off from school, day care or work

Data for this outcome was only available from one study, where the number of participants who were absent from school, day care or work due to colds were measured (Rerksupapphol, 2012). The outcome is therefore only from this study, with 40 participants for probiotics group and 40 participants for placebo group. Of the 40 participants in the placebo group, 14 participants had experienced cold related absence, only two participants from the probiotic group experienced absence due to colds. Table 8 and table 9 shows the crosstabulation of results and chi-square test respectively that were performed to analyse the data. 
Table 8: Intervention and number of participants who experienced cold related absence

\begin{tabular}{|c|c|c|c|c|c|}
\hline & & & \multicolumn{2}{|c|}{ Absence } & \multirow[b]{2}{*}{ Total } \\
\hline & & & No & Yes & \\
\hline \multirow[t]{4}{*}{ Intervention } & Placebo & $\underline{\text { Count }}$ & $\underline{26}$ & 14 & 40 \\
\hline & & Expected Count & 32.0 & 8.0 & 40.0 \\
\hline & Probiotic & $\underline{\text { Count }}$ & $\underline{38}$ & 2 & 40 \\
\hline & & Expected Count & 32.0 & 8.0 & 40.0 \\
\hline \multirow[t]{2}{*}{ Total } & & $\underline{\text { Count }}$ & $\underline{64}$ & 16 & 80 \\
\hline & & Expected Count 64.0 & 16.0 & 80.0 & \\
\hline
\end{tabular}

Table 9: Chi-square test for intervention and cold related absence

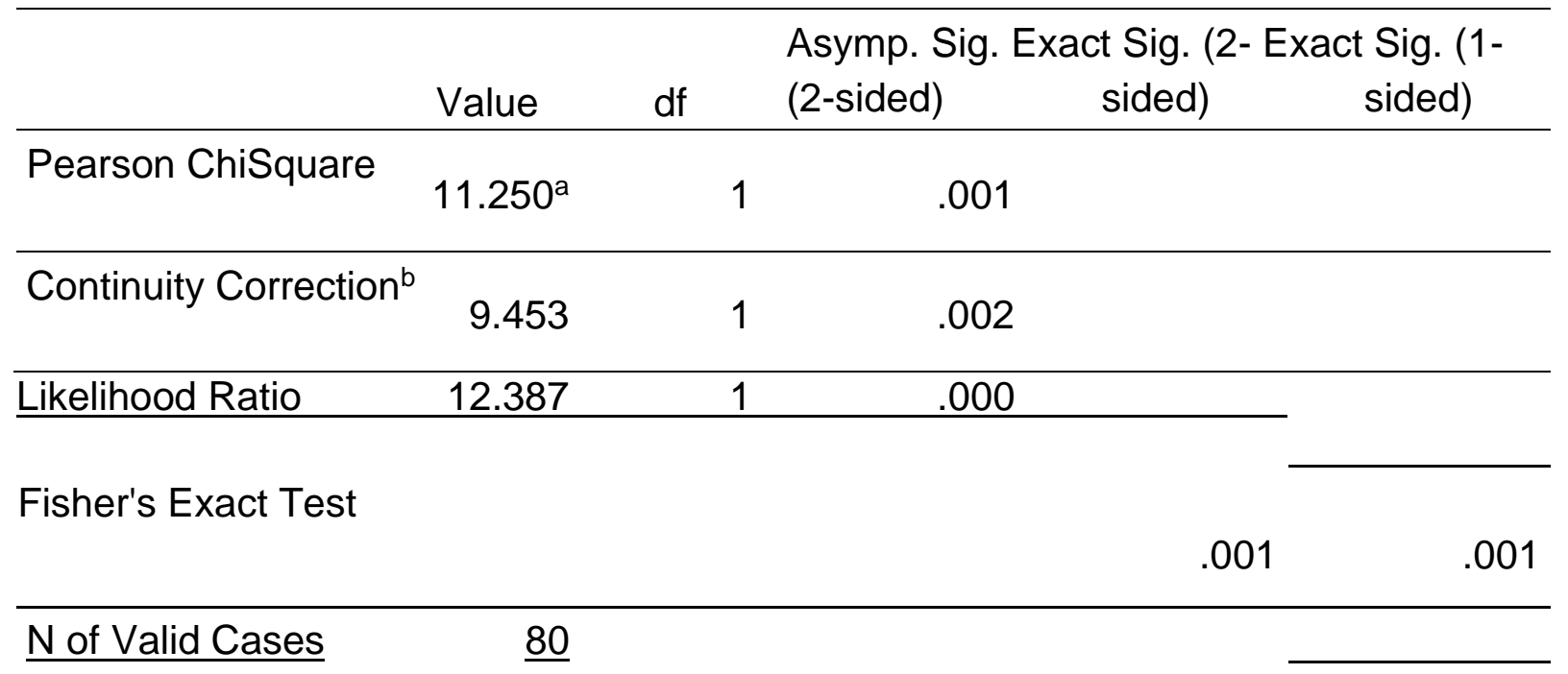

a. 0 cells $(0.0 \%)$ have expected count less than 5 . The minimum expected count is 8.00 . 
Table 8 shows the number of participants who experienced cold related absence for placebo group is higher than the expected count by six individuals, while the number for the probiotic group is lower by six individuals. This shows that probiotics is better than placebo in reducing the number of absents due to cold. The chi-square $p$-value obtained is $p=0.001<0.05$. The null hypothesis is rejected, this shows there is significant difference between intervention and cold-related absence. Absence from school, day care or work due to URTI was not reported by any other included trials in this meta-analysis, due to the small sample size the outcome results are of low quality, more studies must be done on this aspect for a more conclusive outcome. 


\subsection{Conclusion}

In this meta-analysis and systematic review, probiotics were found to be better compared to placebo in reducing acute upper respiratory tract infections experienced by participants. Probiotics were also shown to lower the mean duration of acute URTI episodes and decrease antibiotic use in participants. One study also measured the absence from school, day care or work due to URTI, results were found to be statistically significant and that probiotic was beneficial in preventing absence due to URTI. Study data analysed also show that adverse side effects such as vomiting, diarrhoea, flatulence, bowel irritation and nausea were minor and not statistically significant to intervention. However, due to the small number of trials included for certain outcome measures and possible bias, the results from this review should be taken mindfully. Furthermore, many included trials in this review had not a detailed account of the methodology used in performing the study. Some studies also had a primary outcome measure that varied from the primary focus of this meta-analysis, which could introduce bias. Future research on probiotics in a randomised controlled trial can benefit from better quality blinding of participant allocation to improve the quality of results. Secondly, assessing similar biomarkers or outcome measures for more precise analyses when pooling multiple studies, such as number of individuals who experienced acute URTI or mean duration of one acute URTI episode.

DOI: 10.13140/RG.2.2.14952.08962 


\subsection{References to included studies}

Adorno, M., Garbee, D. and Marix, M. (2016) Improving Literature Searches. Clinical Nurse Specialist [online]. 30 (2), pp. 74-80. [Accessed 24 January 2018].

Bernaola Aponte, G., Bada Mancilla, C., Carreazo, N. and Rojas Galarza, R. (2013) Probiotics for treating persistent diarrhoea in children. Cochrane Database of Systematic Reviews [online]. [Accessed 27 February 2018].

Bienenstock, J., Gibson, G., Klaenhammer, T., Walker, W. \& Neish, A. (2013) New insights into probiotic mechanisms. Gut Microbes [online]. 4 (2), pp. 94-100. [Accessed 25 January 2018].

Bienenstock, J., Gibson, G., Klaenhammer, T., Walker, W. and Neish, A. (2013) New insights into probiotic mechanisms. Gut Microbes [online]. 4 (2), pp. 94-100. [Accessed 7 February 2018].

Boesten, R. and de Vos, W. (2008) Interactomics in the Human Intestine. Journal of Clinical Gastroenterology [online]. 42pp. S163-S167. [Accessed 3 February 2018].

Butel, M. (2014) Probiotics, gut microbiota and health. Médecine et Maladies Infectieuses [online]. 44 (1), pp. 1-8. [Accessed 3 February 2018].

Clemente, J., Ursell, L., Parfrey, L. and Knight, R. (2012) The Impact of the Gut Microbiota on Human Health: An Integrative View. Cell [online]. 148 (6), pp. 1258-1270. [Accessed 13 February 2018].

Corthésy, B. (2013) Multi-Faceted Functions of Secretory $\lg A$ at Mucosal Surfaces. Frontiers in Immunology [online]. 4. [Accessed 13 February 2018].

Fujita, R., limuro, S., Shinozaki, T., Sakamaki, K., Uemura, Y., Takeuchi, A., Matsuyama, Y. and Ohashi, Y. (2013) Decreased duration of acute upper respiratory tract infections with daily intake of fermented milk: A multicenter, double-blinded, randomized comparative study in users of day care facilities for the elderly population. American Journal of Infection Control [online]. 41 (12), pp. 1231-1235. [Accessed 27 February 2018].

Gupta, V. and Garg, R. (2009) Probiotics. Indian Journal of Medical Microbiology [online]. 
27 (3), pp. 202. [Accessed 25 January 2018].

Hampton, S. and Parker, J. (2011) Collaboration and Productivity in Scientific Synthesis. BioScience [online]. 61 (11), pp. 900-910. [Accessed 1 January 2018].

Hatakka, K. (2001) Effect of long term consumption of probiotic milk on infections in children attending day care centres: double blind, randomised. BMJ [online]. 322 (7298), pp. 1327-1327. [Accessed 26 February 2018].

Hatakka, K., Blomgren, K., Pohjavuori, S., Kaijalainen, T., Poussa, T., Leinonen, M., Korpela, R. and Pitkäranta, A. (2007) Treatment of acute otitis media with probiotics in otitis-prone children-A double-blind, placebo-controlled randomised study. Clinical Nutrition [online]. 26 (3), pp. 314-321. [Accessed 27 February 2018].

Hemaiswarya, S., Raja, R., Ravikumar, R. and Carvalho, I. (2013) Mechanism of action of probiotics. Brazilian Archives of Biology and Technology [online]. 56 (1), pp. 113-119. [Accessed 7 February 2018].

Hojsak, I., Snovak, N., Abdović, S., Szajewska, H., Mišak, Z. and Kolaček, S. (2010) Lactobacillus $G G$ in the prevention of gastrointestinal and respiratory tract infections in children who attend day care centers: A randomized, double-blind, placebo-controlled trial. Clinical Nutrition [online]. 29 (3), pp. 312-316. [Accessed 27 February 2018].

K Gogineni, V. and Morrow, L. (2013) Probiotics: Mechanisms of Action and Clinical Applications. Journal of Probiotics \& Health [online]. 01 (01), . [Accessed 25 January 2018].

Koch, S. and Nusrat, A. (2012) The Life and Death of Epithelia During Inflammation: Lessons Learned from the Gut. Annual Review of Pathology: Mechanisms of Disease [online]. 7 (1), pp. 35-60. [Accessed 6 February 2018].

Lahtinen, S. (2012) Probiotic viability - does it matter?. Microbial Ecology in Health \& Disease [online]. 23 (0). [Accessed 31 January 2018].

Leite, A., Miguel, M., Peixoto, R., Ruas-Madiedo, P., Paschoalin, V., Mayo, B. and Delgado, S. (2015) Probiotic potential of selected lactic acid bacteria strains isolated from Brazilian kefir grains. Journal of Dairy Science [online]. 98 (6), pp. 3622-3632. [Accessed 25 January 2018]. 
Maggio, L., Tannery, N. and Kanter, S. (2011) Reproducibility of Literature Search Reporting in Medical Education Reviews. Academic Medicine [online]. 86 (8), pp. 10491054. [Accessed 24 January 2018].

Maier, H. (2013) What constitutes a good literature review and why does its quality matter?. Environmental Modelling \& Software [online]. 43pp. 3-4. [Accessed 8 January 2018].

Paul, W. (2011) Bridging Innate and Adaptive Immunity. Cell [online]. 147 (6), pp. 12121215. [Accessed 25 January 2018].

Pautasso, M. (2010) Worsening file-drawer problem in the abstracts of natural, medical and social science databases. Scientometrics [online]. 85 (1), pp. 193-202. [Accessed 8 January 2018].

Pommerville, J., Alcamo, I. and Alcamo, I. (2013) Alcamo's fundamentals of microbiology [online]. 2nd edition. Sudbury, MA: Jones \& Bartlett Learning. [Accessed 6 Febuary 2018].

Prete, R., Tofalo, R., Federici, E., Ciarrocchi, A., Cenci, G. and Corsetti, A. (2017) FoodAssociated Lactobacillus plantarum and Yeasts Inhibit the Genotoxic Effect of 4Nitroquinoline-1-Oxide. Frontiers in Microbiology [online]. 8. [Accessed 25 January 2018].

Rapple, C. (2011) The role of the critical review article in alleviating information overload. Annual Reviews White Paper [online]. 24 (3), pp. 237-238. [Accessed 8 January 2018].

Rautava, S., Salminen, S. and Isolauri, E. (2008) Specific probiotics in reducing the risk of acute infections in infancy - a randomised, double-blind, placebo-controlled study. British Journal of Nutrition [online]. 101 (11), pp. 1722. [Accessed 27 February 2018]. 


\subsection{References}

Rerksuppaphol, S. and Rerksuppaphol, L. (2012) Randomized controlled trial of probiotics to reduce common cold in schoolchildren. Pediatrics International [online]. 54 (5), pp. 682-687. [Accessed 27 February 2018].

Ritchie, M. and Romanuk, T. (2012) A Meta-Analysis of Probiotic Efficacy for Gastrointestinal Diseases. PLoS ONE [online]. 7 (4), pp. e34938. [Accessed 31 January 2018].

Rol, N., Favre, L., Benyacoub, J. and Corthésy, B. (2012) The Role of Secretory Immunoglobulin A in the Natural Sensing of Commensal Bacteria by Mouse Peyer's Patch Dendritic Cells. Journal of Biological Chemistry [online]. 287 (47), pp. 40074-40082. [Accessed 7 February 2018].

Russell, P. (2009) Physiological and Functional Properties of Probiotics (2008) - by International Dairy Federation. International Journal of Dairy Technology [online]. 62 (2), pp. 290-291. [Accessed 29 January 2018].

Sanders, M. (2008) Probiotics: definition, sources, selection, and uses. Clinical infectious Diseases [online]. 46 (2), pp. S58-S61. [Accessed 31 January 2018].

Sedgwick, P. (2013) Snowball sampling. BMJ. 347 [online]. (dec20 2), pp. f7511-f7511. [Accessed 24 January 2018].

Smith, T., Rigassio-Radler, D., Denmark, R., Haley, T. and Touger-Decker, R. (2012) Effect of Lactobacillus rhamnosus $L G G \otimes$ and Bifidobacterium animalis ssp. lactis BB-12® on health-related quality of life in college students affected by upper respiratory infections. British Journal of Nutrition [online]. 109 (11), pp. 1999-2007. [Accessed 27 February 2018].

Tobita, K., Yanaka, H. and Otani, H. (2010) Lactobacillus crispatus KT-11 Enhances Intestinal Immune Functions in $\mathrm{C} 3 \mathrm{H} / \mathrm{HeN}$ Mice. Journal of Nutritional Science and Vitaminology [online]. 56 (6), pp. 441-445. [Accessed 13 February 2018].

Xiong, Y., Wu, F., Wang, J., Li, J. and Kong, L. (2012) Attenuation of airway hyperreactivity and $T$ helper cell type 2 responses by coumarins from Peucedanum 
praeruptorum Dunn in a murine model of allergic airway inflammation. Journal of Ethnopharmacology [online]. 141 (1), pp. 314-321. [Accessed 3 February 2018].

Ya, T., Zhang, Q., Chu, F., Merritt, J., Bilige, M., Sun, T., Du, R. and Zhang, H. (2008) Immunological evaluation of Lactobacillus casei Zhang: a newly isolated strain from koumiss in Inner Mongolia, China. BMC Immunology [online]. 9 (1), pp. 68. [Accessed 13 February 2018].

Yasui, H., Kiyoshima, J. and Hori, T. (1999) Reduction of Influenza Virus Titer and Protection against Influenza Virus Infection in Infant Mice Fed Lactobacillus casei Shirota. Clinical and Vaccine Immunology [online]. 11 (4), pp. 675-679. [Accessed 27 February 2018]. 


\section{APPENDIX A}

The following key words were used in the search strategy to search ScienceDirect and Springer.

1. Probiotics

2. Supplements

3. Common cold

4. Upper respiratory tract infections

5. Infections

6. Upper respiratory infections

7. Lactobacillus

8. Bifidobacterium

9. URTI

10. Immune system

11. Pharyngitis

12. Laryngitis

13. Fever

14. Otitis media

15. Tonsillitis

16. Streptococcus

DOI: 10.13140/RG.2.2.14952.08962 